\title{
Analyser le discours écrit : les configurations relationnelles en français contemporain
}

\author{
Giovanni Agresti \\ Université de Teramo \\ giagresti@yahoo.it
}

\section{Plan de l'article}

Dans cet article nous proposons une démarche d'analyse du discours, notamment écrit, fondée sur le critère de la relationalité textuelle ${ }^{1}$. Pour ce faire, nous reviendrons d'abord ( $\$ 2$ ) sur la nature du discours en général, afin d'établir une terminologie à haut rendement; ensuite, sur ce soubassement conceptuel nous introduirons ( $(3)$ la notion de «configuration relationnelle », qui nous permettra, tant au niveau de la macrostructure $(\S 3.1)$ qu'au niveau de la microstructure (\$ 3.2) d'asseoir quelques différences foncières entre la dynamique du discours oral et celle du discours écrit - différences découlant principalement de la variation du statut de l'interaction. Nous tâcherons enfin $(\S 4)$ de proposer l'analyse textuelle configurationnelle d'un texte écrit.

\section{Nature du discours}

Pour sonder la nature du discours, quatre éléments nous paraissent incontournables ${ }^{2}$, en ce qu'ils sont impliqués les uns par les autres :

- le sujet (S), auteur du texte (qui est en quelque sorte son prolongement) fabriqué à partir d'une visée de reconfiguration du maillage social ;

- le maillage social $(\mathrm{M})$, à savoir l'autre (individuel ou collectif) qui, par sa nature éminemment relationnelle, est la cible de la visée du sujet véhiculée par le texte;

- le texte (T), actualisant la visée du sujet qui veut, à travers lui, reconfigurer le maillage social ;

- la visée (V), qui est l'instance qui pousse le sujet à fabriquer un texte (et qui normalement devrait infléchir sa réalisation) en fonction de sa réception de la part du maillage social.

Chacun de ces quatre éléments entretenant des rapports nécessaires avec tous les autres, on ne saurait les définir en dehors de cette réciprocité : à bien y voir, ils sont à la fois sources et cibles les uns des autres. Mieux : ils sont les uns des composantes des autres. Ce qui nous amène à représenter la relation linguistique intersubjective (désormais RLI) de la manière suivante : 
Tableau 1 : la RLI

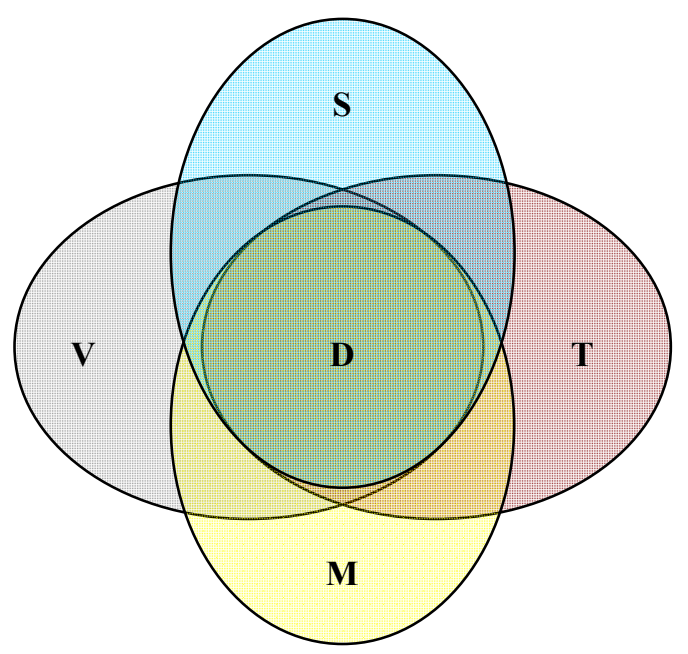

Au cœur de ce schéma, un élément supplémentaire surgit de la synthèse des quatre premiers : sous le graphème $\mathrm{D}$, le «discours » se pose en tant que «texte » étoffé par rapport à $\mathrm{T}$, à savoir le simple support linguistique.

On peut ainsi lire notre tableau de deux manières : a) depuis le point de vue de $\mathrm{S}$ bâtissant son $\mathrm{D}$, ce pour quoi $\mathrm{S}$ va mobiliser ses compétences pour parvenir à $\mathrm{T}$ en raison de $\mathrm{V}$ afin d'atteindre $\mathrm{M}-$; ou bien b) depuis le point de vue de l'analyse, qui à partir de $\mathrm{T}$ (c'est le seul élément mesurable d'une manière objective) tâche de saisir $\mathrm{D}$ en remontant à $\mathrm{S}, \mathrm{V}, \mathrm{M}$. C'est cette perspective de décryptage que nous tâcherons de développer dans la présente étude.

La différence entre $\mathrm{T}$ et $\mathrm{D}$ est capitale et subtile à la fois, car elle ne concerne guère l'objet textuel en lui-même, mais plutôt notre façon de l'appréhender. Elle ne relève donc pas d'une véritable dissociation notionnelle (Adam, Grize \& Bouacha, 2004), mais plutôt d'un déplacement et d'une transformation du point et de la qualité de l'observation. Tout texte, aussi bien écrit qu'oral, se présente comme une surface pouvant à la fois cacher et révéler ses profondeurs - que nous pouvons, en gros, ramener à la dialectique des trois autres éléments $(\mathrm{S}, \mathrm{V}, \mathrm{M})$. Cette surface linguistique est en effet opaque en même temps que perçable, en ce qu'elle est, de ces éléments en jeu, la projection, le reflet, la réfraction. Bref, le discours (D) est le texte (T) dont on essaiera de saisir les rapports entre ses différentes composantes ${ }^{3}$.

Ce que nous venons de dire a de lourdes conséquences, que l'on connaît bien : D est un produit conscient de $\mathrm{S}$ en même temps qu'il échappe, en partie, à son contrôle, car $\mathrm{S}$ n'en est nullement le maître absolu' ${ }^{4}$ Le sujet est aussi l'autre (M) qu'il a pour cible, et cet autre co-construit le discours ${ }^{5}$. Or le maillage social est un tissu conjonctif multidimensionnel : la co-construction de D dépend non seulement de l'autre physiquement présent, mais également de l'autre physiquement absent, ainsi que de cet autre collectif que d'aucuns appellent la doxa, et que nous appellerons plutôt, d'une manière plus générale et systématique, l'autre latent. D'où un triplement parallèle du statut de l'interaction.

Comme nous le verrons plus loin, ces trois formes d'autre coexistent et exercent de manières diverses leur conditionnement sur la construction de D, puisqu'elles habitent, parfois hantent, S. Car celui-ci, lorsqu'il est un individu et non pas un sujet purement textuel, est à bien des égards un "être de langage » (Lafont, 2004), à savoir un être relationnel, et ces relations construisent fatalement son identité. 
En effet, s'il est vraisemblable que chaque texte $\left(T_{1}, T_{2}, T_{3} \ldots\right)$ nait en fonction de visées particulières $\left(\mathrm{V}_{1}, \mathrm{~V}_{2}, \mathrm{~V}_{3} \ldots\right)$, à un niveau plus abstrait on peut émettre l'hypothèse que celles-ci concourent vers un même but profond, voire inconscient. D'après (Vygotskij, 1934), ce but serait l'identité de pensée entre les interlocuteurs, soit $\mathrm{S} \equiv \mathrm{M}$ - à terme une weltanschauung partagée. Il y a là l'ébauche d'une idée qui nous paraît féconde : la construction de $\mathrm{D}$ en fonction de la construction de l'identité collective. Cette idée, qui doublerait sub specie linguae l'approche interactionniste des sciences sociales (Charaudeau Maingueneau, 2002 : 319-320) est néanmoins trop générale (donc difficilement systématisable) pour être développée ici.

Quoi qu'il en soit, une observation, à l'air banal, s'impose : de même que le Tableau 1 le met en évidence, un texte, n'importe quel texte, est d'abord et surtout un outil de mise en relation, de liage. Même un panneau indicateur, texte minimal où souvent le langage déchoit en code, n'échappe pas à ce principe : bien qu'impersonnel, il met l'observateur en relation avec un réservoir d'informations - plus ou moins riche, général ou spécifique, opaque ou transparent. L'accès à ces informations contribue à positionner (ou à repositionner) cet observateur dans le maillage social.

Qui plus est, si D est à la fois, en aval, outil pour, et, en amont, résultat d'une mise en relation, l'analyse de D peut substantiellement revenir à celle de la relationalité du texte. On n'est pas loin de la notion de « texte comme unité compositionnelle et configurationnelle » (Adam, 2005). Toutes différences gardées, (nous nous intéressons moins de «sens» que de «relation») nous appellerons analyse configurationnelle l'opération qui consiste à faire ressortir l'architecture (ou configuration) relationnelle cachée dans les profondeurs du texte. En d'autres termes: dans le maillage du discours, de la macrostructure à la microstructure, nous tâcherons de dessiner les dynamiques ou configurations sociales (au sens large du mot) sous-jacentes par rapport à la surface textuelle (qui les actualise), c'est-àdire par rapport aux configurations linguistiques. C'est peut-être là, dans ce décalage, et entre ces deux éclairages, la loge de l'idéologie, notamment dans certaines classes de discours (le discours « politique » par exemple, où $\mathrm{S}$ vise, si possible plus qu'ailleurs, à fabriquer le consensus moyennant le déploiement de son éthos) ${ }^{6}$.

\section{Nature des configurations relationnelles}

\subsection{Macrostructure}

A bien y voir, dans la langue tout a l'air d'être plus ou moins « relationnel », de même que tout a l'air plus ou moins « fonctionnel». Pour surmonter ce flou conceptuel, il nous faut articuler ultérieurement notre terminologie afin de décrire de manière pertinente ces dynamiques relationnelles.

Pour ce faire, revenons d'abord sur la nature de S qui, s'il n'est nullement le maitre absolu du discours, en est sans aucun doute l'acteur principal. $\mathrm{S}$ peut être personnel $\left(\mathrm{S}_{\mathrm{p}}\right)$ (sujet réel et textuel), indéfini $\left(\mathrm{S}_{\mathrm{i}}\right)$ (sujet textuel) ou impersonnel $\left(\mathrm{S}_{0}\right)$ (sujet textuel). Comme nous l'avons dit plus haut, il est conditionné en permanence par un appel d'autre : un savoir diffus (latent), un groupe défini ou bien une personne connue (présente ou absente).

Un exemple concernant l'écrit nous paraît remarquable : c'est le cas, à l'opposé du panneau indicateur que nous venons d'évoquer, de la lettre manuscrite traçant une relation sentimentale d'individu à individu, soit le «discours amoureux » : ce texte, fort personnel jusque dans l'outil d'écriture choisi, mobilise et touche la corde sensible du sujet : l'autre y est bien perceptible tant du côté de l'émetteur (agent) que du côté du récepteur (co-agent), jusqu'à imposer sa présence par-delà son absence physique.

Pourtant, en analysant la texture de cette lettre passionnée, on ne saurait exclure l'action cachée d'autres sujets : notamment le sujet collectif, qui valide et éventuellement limite (autorise) l'expression personnelle par une espèce d'argument d'autorité, à savoir un précédent, bref un modèle linguistique (lexical, morphologique, syntaxique ou stylistique) censé protéger en quelque sorte l'intimité du sujet des risques d'une expression trop originale, à savoir personnelle, qui l'exposerait excessivement à 
l'autre. Les adolescents en quête d'identité exhibent ainsi leur ego à travers des hypergraphies ou des hyperécritures sur les murs des villes tout en le mettant à l'abri d'une vulnérabilité à laquelle ils ne sont pas prêts par le biais du caractère stéréotypé de ces expressions mêmes : «I love u 4 ever », « Je t'm »" «T.V.B. »... ou n'importe quelle citation. Après tout, c'est toujours le langage des fleurs : car finalement personne n'échappe à l'influence protectrice du moule. C'est l'autre ou la société latente qui exerce cette influence, qui bien évidemment peut varier beaucoup d'un individu à l'autre ${ }^{8}$.

Les discours, de l'oral à l'écrit, en passant par des formes mixtes, qui sont légion de nos jours (et dont, faute d'espace, on fera l'économie dans le présent article), reçoivent et enregistrent ainsi des instances différentes. Sans doute, pourrait-on évoquer des "points de vue », mais nous préférons parler de trois forces jouant dans la texture langagière et au-dessous d'elle : autre présent $\left(\mathrm{M}_{\mathrm{p}}\right)$, absent $\left(\mathrm{M}_{\mathrm{a}}\right)$ ou latent $\left(\mathrm{M}_{1}\right)$. Ces trois forces ne fonctionnent presque jamais en solo, mais plutôt simultanément. Pour ce qui est de l'oral, deux exemples (dont le deuxième se fonde sur un témoignage réel), que nous avons ailleurs développés (Agresti, 2008 : 102-104), nous paraissent particulièrement parlants : le discours funèbre et le meeting en langue minoritaire :

- dans le premier cas, le parent ou l'ami $\left(\mathrm{S}_{\mathrm{p}}\right)$ du défunt prononce bien un discours (D) adressé aux gens présents dans l'église $\left(M_{p}\right)$, mais le pivot de ce discours est l'autre absent $\left(M_{a}\right)$, que les mots de $S$ ont tendance $(\mathrm{V})$ à rendre présent. L'hommage de mémoire est dû aux personnes disparues, car leur absence étant irréversible, seule la mémoire partagée peut «ramener», ne serait-ce que psychologiquement, l'autre au présent. Mais D est également conditionné par la troisième dimension du maillage social $\left(\mathrm{M}_{1}\right)$ : la société latente, à savoir les conventions, la praxis, bref le style reçu qui infléchit parfois lourdement ce type de discours (un véritable « texte spécialisé ») en contrebalançant la forte subjectivité (émotionnelle) que l'on peut évidemment attribuer à $\mathrm{S}_{\mathrm{p}}$;

- dans le deuxième cas, le meeting en langue minoritaire, l'emplacement valorisant d'une langue historiquement dominée par la langue d'Etat, dans un cadre marqué par une sorte de rituel collectif (le meeting), s'est révélé D même indépendamment de T ! Preuve en est le fait qu'il a déclenché très rapidement, chez une jeune spectatrice (autre présent, $M_{p}$ ), un choc affectif court-circuitant la mémoire de langue (autre latent, $\mathrm{M}_{1}$ ), sans doute dévalorisante, du récepteur, rétablissant ainsi du coup des liens familiaux (autre absent, $\mathrm{M}_{\mathrm{a}}$ ) (c'était la langue de ses «vieux ») chez cette fille.

Ce raisonnement et ces exemples nous permettent de revenir sur l'élément qui joint, par $\mathrm{T}$ et en amont de la fabrication de T, S à M, à savoir la visée (V). Jusqu'ici, nous n'avons parlé que d'un concept, mais on peut supposer que V est pour le moins distribuée (parasitée ?) sur (par) plusieurs niveaux. Au moins deux : celui de l'autre présent, et celui de l'autre absent. On peut ainsi supposer une V davantage directe et une $\mathrm{V}$ seconde, moins directe. Mais cela dépend, évidemment, du contexte dans lequel se déroule la $\mathrm{RLI}$, ainsi que du statut (par exemple, affectif) de la relation $\mathrm{S} \sim \mathrm{M}$.

Ces trois forces (présence, absence, latence) ainsi que ces trois formes du sujet (personnel, indéfini, impersonnel) vont être les fondements de notre approche configurationnelle, prise en tant qu'analyse des mises en relation, via $\mathrm{D}$, entre éléments sources et éléments cibles. Ces «mises en relation », nous les appellerons «configurations relationnelles (macrostructurales)» ou plus simplement «châssis relationnels». Quelle est leur nature? Quel est leur nombre? Nous proposons que, tous textes confondus (écrits et oraux), les châssis soient établis à partir du tableau suivant (le rouge indique un trait propre aux textes écrits, le style diagonal indique un trait propre aux textes oraux) : 
Tableau 2 : les châssis relationnels

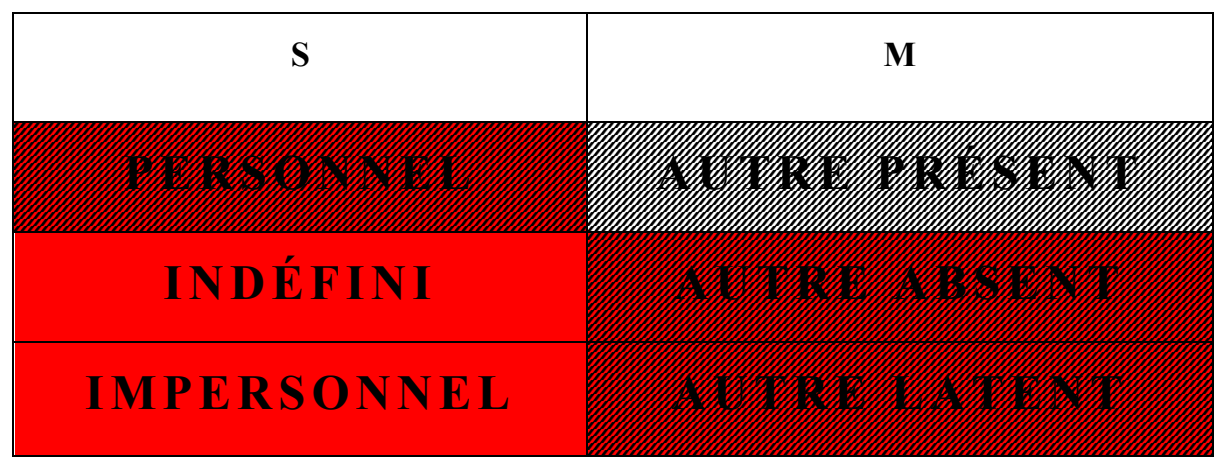

$\mathrm{Si}$, dans les textes écrits, S peut être personnel (c'est un sujet bien défini qui s'exprime), indéfini (c'est un sujet anonyme ou non parfaitement repérable) ou impersonnel (c'est une voix sans sujet, comme notre panneau indicateur), $\mathrm{M}$ ne sera qu'absent ou latent, à quelques rares exceptions près ; vice-versa, dans les textes oraux (interactionnels), malgré ses déguisements et ses jeux d'esquive, seul S personnel est admissible, alors que $\mathrm{M}$ pourra être à la fois présent, absent ou latent ${ }^{9}$. A partir de là, une linguistique configurationnelle du discours écrit par rapport à une linguistique configurationnelle du discours oral paraît envisageable. Voyons maintenant en détail quelles sont ces relations possibles entre $\mathrm{S}$ et $\mathrm{M}$ :

Tableau 3 : châssis relationnels et articulations textuelles

\begin{tabular}{|c|c|c|}
\hline Type de relation & Textes oraux & Textes écrits \\
\hline $\begin{array}{l}\text { Sujet présent }\left(\mathrm{S}_{\mathrm{p}}\right) \text { en } \\
\text { relation avec un } \\
\text { autre présent }\left(\mathrm{M}_{\mathrm{p}}\right)\end{array}$ & $\begin{array}{l}\text { Il s'agit de l'interaction orale, c'est- } \\
\text { à-dire en présence. S fonde le critère } \\
\text { de réalité (Lafont, } 2004: 89-92 \text { ). }\end{array}$ & $\begin{array}{l}\text { Ce type de relation n'est normalement } \\
\text { pas possible }\end{array}$ \\
\hline $\begin{array}{l}\text { Sujet présent }\left(\mathrm{S}_{\mathrm{p}}\right) \text { en } \\
\text { relation avec un } \\
\text { autre absent }\left(\mathrm{M}_{\mathrm{a}}\right)\end{array}$ & $\begin{array}{l}\text { En amont de la relation en présence, } \\
M_{a} \text { pousse } S_{p} \text { à fabriquer un discours } \\
\text { destiné à } M_{p} \text {. Mais } M_{a} \text { peut } \\
\text { également être la forme, le modèle } \\
\text { d'après lequel le sujet voudrait } \\
\text { reconfigurer } M \text { par la RLI. }\end{array}$ & $\begin{array}{l}\text { Le sujet, défini, adresse un texte à un } \\
\text { destinataire fatalement distant, soit-il } \\
\text { connu ou inconnu. La presque totalité } \\
\text { des textes signés ou d'auteur relève de } \\
\text { cette configuration. }\end{array}$ \\
\hline $\begin{array}{l}\text { Sujet présent }\left(\mathrm{S}_{\mathrm{p}}\right) \text { en } \\
\text { relation avec un } \\
\text { autre latent }\left(\mathrm{M}_{1}\right)\end{array}$ & $\begin{array}{l}\text { Au-dessous de la relation en } \\
\text { présence, ce châssis donne accès au } \\
\text { matériau exploité par } \mathrm{S}_{\mathrm{p}} \text { pour } \\
\text { actualiser } \mathrm{M}_{\mathrm{a}} \text { La relation est en effet } \\
\text { intimement liée à la langue } \\
\text { employée, et au réservoir } \\
\text { d'inconscient que le sujet partage } \\
\text { avec les membres de sa communauté } \\
\text { (M). Il s'agit par là d'une relation } \\
\text { profonde (le "plaisir de la langue ", } \\
\text { par exemple) dont le sujet n'a } \\
\text { conscience que par auto-observation. }\end{array}$ & $\begin{array}{l}\text { La relation est intimement liée à la } \\
\text { langue employée, et au réservoir } \\
\text { d'inconscient que le sujet partage avec } \\
\text { les membres de sa communauté (M). } \\
\text { Il s'agit par là d'une relation profonde } \\
\text { (le " plaisir de la langue ", par } \\
\text { exemple) dont le sujet n'a conscience } \\
\text { que par auto-observation. Sans doute, } \\
\text { notamment pour ce qui est de textes } \\
\text { ciselés, cette auto-observation } \\
\text { fonctionne-t-elle plus à l'écrit qu'à } \\
\text { l'oral. }\end{array}$ \\
\hline $\begin{array}{l}\text { Sujet indéfini }\left(\mathrm{S}_{\mathrm{i}}\right) \\
\text { en relation avec un } \\
\text { autre présent }\left(\mathrm{M}_{\mathrm{p}}\right)\end{array}$ & $\begin{array}{l}\text { Ce type de relation n'est } \\
\text { normalement pas possible }\end{array}$ & $\begin{array}{l}\text { Ce type de relation n'est normalement } \\
\text { pas possible }\end{array}$ \\
\hline
\end{tabular}




\begin{tabular}{|c|c|c|}
\hline $\begin{array}{l}\text { Sujet indéfini }\left(\mathrm{S}_{\mathrm{i}}\right) \\
\text { en relation avec un } \\
\text { autre absent }\left(\mathrm{M}_{\mathrm{a}}\right)\end{array}$ & $\begin{array}{l}\text { Ce type de relation n'est } \\
\text { normalement pas possible }\end{array}$ & $\begin{array}{l}\text { Le statut du sujet (qui peut être en fait } \\
\text { une rédaction) a tendance à se } \\
\text { confondre avec celui de son public. Ce } \\
\text { type de relation paraît } \\
\text { particulièrement propice à véhiculer } \\
\text { des idées reçues, ou des idées } \\
\text { nouvelles aisément «recevables »par } \\
\text { le lecteur. Le langage de la publicité } \\
\text { pourrait, en gros, relever de cette } \\
\text { configuration. }\end{array}$ \\
\hline $\begin{array}{l}\text { Sujet indéfini }\left(\mathrm{S}_{\mathrm{i}}\right) \\
\text { en relation avec un } \\
\text { autre latent }\left(\mathrm{M}_{1}\right)\end{array}$ & $\begin{array}{l}\text { Ce type de relation n'est } \\
\text { normalement pas possible }\end{array}$ & $\begin{array}{l}\text { Le texte garde bien les signes de la } \\
\text { fabrication de la part d'un sujet, sans } \\
\text { pour autant qu'il soit possible de } \\
\text { remonter à l'identité de celui-ci. Par } \\
\text { affadissement du sens d'abord, du } \\
\text { signifié ensuite, dû à l'érosion } \\
\text { diachronique et aux multiples } \\
\text { déplacements de D, l'autre absent a } \\
\text { déchu en autre absent second, soit } \\
\text { latent: on retrouve ce châssis à } \\
\text { l'œuvre, par exemple, dans de vieilles } \\
\text { inscriptions partiellement intelligibles, } \\
\text { qui ne renvoient plus directement à un } \\
\text { autre absent, à savoir à un public, mais } \\
\text { plutôt à une dimension profonde, } \\
\text { latente, où un autre public, } \\
\text { éventuellement, ne reconnaîtra plus } \\
\text { que d'autres sens et signifiés (la } \\
\text { consistance du temps, la permanence } \\
\text { de la trace signifiante, l'âge des } \\
\text { langues, etc.) On voit bien qu'il s'agit } \\
\text { là d'une re-construction de D, où le } \\
\text { temps (et plus en général la } \\
\text { «distance ») joue un rôle décisif. }\end{array}$ \\
\hline $\begin{array}{l}\text { Sujet impersonnel } \\
\left(\mathrm{S}_{0}\right) \text { en relation avec } \\
\text { un autre présent }\left(\mathrm{M}_{\mathrm{p}}\right)\end{array}$ & $\begin{array}{l}\text { Ce type de relation n'est } \\
\text { normalement pas possible }\end{array}$ & $\begin{array}{l}\text { Ce type de relation n'est normalement } \\
\text { pas possible }\end{array}$ \\
\hline $\begin{array}{l}\text { Sujet impersonnel } \\
\left(\mathrm{S}_{0}\right) \text { en relation avec } \\
\text { un autre absent }\left(\mathrm{M}_{\mathrm{a}}\right)\end{array}$ & $\begin{array}{l}\text { Ce type de relation n'est } \\
\text { normalement pas possible }\end{array}$ & $\begin{array}{l}\text { Le texte est fabriqué en effaçant } \\
\text { complètement le sujet afin de s'adresser } \\
\text { de manière purement référentielle à un } \\
\text { lecteur. On retrouve ce type de relation } \\
\text { dans les modes d'emploi, les panneaux } \\
\text { indicateurs, les règlements, etc. }\end{array}$ \\
\hline $\begin{array}{l}\text { Sujet impersonnel } \\
\left(\mathrm{S}_{0}\right) \text { en relation avec } \\
\text { un autre latent }\left(\mathrm{M}_{1}\right)\end{array}$ & $\begin{array}{l}\text { Ce type de relation n'est } \\
\text { normalement pas possible }\end{array}$ & $\begin{array}{l}\text { La relation est zéro: } \mathrm{S} \equiv \mathrm{T} \text {, l'autre } \\
\text { n'étant pas identifiable, } \mathrm{V} \text { se replie sur } \\
\text { elle-même : le sujet, impersonnel, est } \\
\text { omniscient et inscrit son dire dans le } \\
\text { système de valeurs même qui structure } \\
\mathrm{M} \text {. Cette configuration régit l'adage, } \\
\text { le proverbe, la maxime } \\
\text { (traditionnelle: à savoir, dont on a } \\
\text { oublié l'auteur). }\end{array}$ \\
\hline
\end{tabular}

Ce tableau mériterait évidemment de longs commentaires. Nous nous bornerons à observer que, vu la mouvance du rapport $\mathrm{S}$ (personnel, indéfini, impersonnel) $\sim \mathrm{M}$ (présent, absent, latent) on ne peut 
nullement, par là, asseoir de taxinomie à haute définition des typologies textuelles. Cependant, ces châssis relationnels fondamentaux peuvent parfois à eux seuls caractériser un type ou une classe de textes. Ou alors ces châssis peuvent être en quelque sorte des horizons discursifs préalables que $\mathrm{S}$, au niveau de la microstructure, pourra exploiter à ses fins. Enfin, ce répertoire suggère une première différence entre la nature des textes oraux et celle des textes écrits.

\subsection{Microstructure}

Notre approche configurationnelle peut s'articuler ultérieurement, au niveau de la microstructure, en prenant en compte de plus près le couple énoncé / énonciation. Ces notions, à la lumière de la démarche illustrée jusqu'ici, acquièrent une valeur précise ${ }^{10}$ :

- par « énonciation » $(E)$, on indique l'action exercée sur M par S : il s'agit de l'in fieri de V ;

- par « énoncé » (E), on indique T, l'in esse de V.

Ainsi, dans les textes oraux, $E$ écrase $\mathrm{E}$ (la mise en relation étant le texte même), alors que dans les textes écrits $\mathrm{E}$ est chronologiquement séparé de $E$. Cette séparation rend possible la mouvance, ou reconfiguration, de $\mathrm{D}$, sa relecture, son évolution par-delà sa cristallisation. La distinction entre $E$ et $\mathrm{E}$ nous permet en effet un dédoublement de l'analyse : d'une part, le plan de E, la fabula, le contenu, l'histoire, par exemple, d'un roman ; et de l'autre, le plan de $E$, à savoir la visée du sujet-auteur, l'action s'exerçant sur son public (M), bref le déploiement de sa stratégie discursive.

Or l'analyse de cette stratégie relève forcément de la microstructure. Les «châssis relationnels fondamentaux » disent une sorte de configuration standard qui demande à être développée au contact de textes bien réels dans leur irréductible diversité, ainsi qu'au contact de langues autres que le français ${ }^{11}$. En amont de cette expérimentation, il est nécessaire de passer de ces "châssis » aux «dispositifs relationnels » (ou « configurations relationnelles microstructurales »), comme on passerait d'une image fixe à une suite de photogrammes donnant l'idée du mouvement. Ce mouvement est d'ailleurs impliqué par la nature même des « forces » évoquées agissant dans D.

Pour ce faire, il faut augmenter la définition de nos configurations relationnelles, en revenant, cette foisci, sur la relation même $\mathrm{S} \sim \mathrm{M}$. L'actualisation de celle-ci laisse des traces au niveau de $\mathrm{T}$ : ce sont les pronoms (personnels, de non-personne, indéfinis et impersonnel) et leur environnement textuel ; aussi, plus largement, d'autres déictiques. S use (consciemment ou pas) de ces véritables dispositifs relationnels pour reconfigurer $\mathrm{M}$, car chaque pronom assoit une configuration, à la fois linguistique et sociale. Cela dit, évidemment, il faudra toujours distinguer, dans l'analyse textuelle, entre le plan de $E$ et celui de E, l'analyse de ce dernier débouchant sur une description de l'organisation de T, celle du premier débouchant sur une herméneutique de $\mathrm{D}$.

Dans le tableau 4 , nous proposons finalement une taxinomie de graphes ${ }^{12}$ censés représenter, tous textes confondus (écrits et oraux), ces configurations relationnelles (linguistiques aussi bien que sociales) au niveau de la microstructure. On y remarquera les trois formes d'actualisation de chaque configuration, à savoir l' «identité » (in esse), lorsqu'une configuration est posée dans D en tant que condition stable, la «construction» ainsi que la «déconstruction » (in fieri), lorsqu'une configuration est en train de se réaliser ou dé-réaliser.

Dans l'analyse textuelle configurationnelle, on distinguera également :

- les valeurs relationnelles intrinsèques de chaque dispositif, c'est-à-dire découlant directement de la structure linguistique du français ;

- les « détours stylistiques », à savoir les effets de discours s'éloignant ou complexifiant ce «degrézéro » ou cette sorte de déterminisme structurel. 
De toute évidence, ces deux phases de l'analyse ne vont pas l'une sans l'autre. Une étude de grand corpus pourra préciser les normes discursives, ainsi qu'une taxinomie des stratégies discursives marquées.

Tableau 4 : les dispositifs relationnels

\begin{tabular}{|c|c|c|}
\hline identité/altérité (être) & construction (devenir) & déconstruction (devenir) \\
\hline $\begin{array}{r}\# \mathbf{j e \#} \\
\text { ego cristallisé de } \mathrm{S}\end{array}$ & $\begin{array}{l}\qquad \begin{array}{l}\# \mathbf{e} \# \uparrow \\
\text { affirmation de l'ego de } \mathrm{S} \text { et sa } \\
\text { séparation }\end{array} \\
\end{array}$ & $\begin{array}{r}\# \mathbf{j e \# \times} \\
\text { crise de l'ego de } \mathrm{S}\end{array}$ \\
\hline 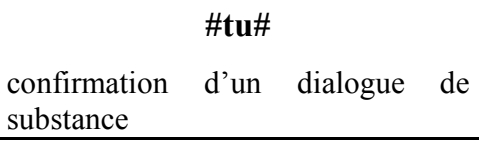 & $\begin{array}{l}\text { \#tu\# } \uparrow \\
\text { dialogue de substance souhaité ou } \\
\text { en cours }\end{array}$ & $\begin{array}{c}\# \text { tu\#× } \\
\text { crise du dialogue de substance }\end{array}$ \\
\hline $\begin{array}{l}\qquad \text { \#vous } \# \\
\text { confirmation d'un dialogue formel } \\
(\text { d'où le «f» en apex })\end{array}$ & \begin{tabular}{|l|}
\multicolumn{1}{|c|}{ \#vous $\# \uparrow$} \\
dialogue formel souhaité ou en \\
cours
\end{tabular} & $\begin{array}{c}\text { \#vous } \# \times \\
\text { crise du dialogue formel }\end{array}$ \\
\hline $\begin{array}{l}\text { \#il-elle\# } \\
\text { confirmation du statut de non- } \\
\text { personne, sujet distant par rapport au } \\
\text { sujet énonçant }\end{array}$ & \begin{tabular}{|l|}
\multicolumn{1}{|c|}{$\#$ il-elle\# $\uparrow$} \\
processus de reconnaissance et \\
d'identification d'un individu
\end{tabular} & $\begin{array}{l}\text { \#il-elle\#× } \\
\text { exclusion d'un individu, non } \\
\text { reconnaissance de son statut, } \\
\text { marginalisation }\end{array}$ \\
\hline $\begin{array}{l}\text { \#nous\# } \\
\text { identité collective, appartenance à } \\
\text { une communauté }\end{array}$ & 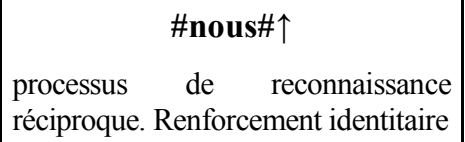 & $\begin{array}{l}\qquad \text { \#nous\#× } \\
\text { crise identitaire, rupture interne à } \\
\text { la communauté }\end{array}$ \\
\hline
\end{tabular}

\begin{tabular}{|c|c|c|}
\hline $\begin{array}{l}\qquad \text { nous }^{\mathbf{f}} \# \\
\text { «nous » formel, confirmation de la } \\
\text { orésence d'un leader au sein de la } \\
\text { ommunauté }\end{array}$ & $\begin{array}{l}\qquad \text { \#nous } \mathbf{f}^{\mathbf{f}} \uparrow \\
\text { processus de centralisation de la } \\
\text { part d'un leader. Construction du } \\
\text { consensus }\end{array}$ & $\begin{array}{l}\text { | } \text { \#nous } \# \times \\
\text { gouvernement central en } \\
\text { discussion, instabilité sociale, } \\
\text { crise des repères communs } \\
\end{array}$ \\
\hline $\begin{array}{l}\text { \#vous\# } \\
\text { confirmation du rapport entre un } \\
\text { ujet (individu ou groupe) central et } \\
\text { in groupe de référence }\end{array}$ & $\begin{array}{l}\text { \#vous\# } \uparrow \\
\text { construction d'un rapport centre- } \\
\text { périphérie, généralisation des } \\
\text { éléments périphériques }\end{array}$ & \begin{tabular}{|lll}
$\mid 4$ & \multicolumn{4}{|c}{ \#vous\#× } \\
conflit direct entre l'autorité \\
$\begin{array}{l}\text { centrale et des éléments } \\
\text { périphériques }\end{array}$ \\
\end{tabular} \\
\hline $\begin{array}{l}\text { \#ils-elles\# } \\
\text { istance d'un groupe de la } \\
\text { ommunauté centrale }\end{array}$ & $\begin{array}{l}\qquad \text { \#ils-elles\# } \uparrow \\
\text { reconnaissance du groupe distant de } \\
\text { la part du groupe central }\end{array}$ & $\begin{array}{l}\text { \#ils-elles\#× } \\
\text { séparation entre la communauté } \\
\text { centrale et le groupe distant }\end{array}$ \\
\hline $\begin{array}{r}\text { \#on\# } \\
\text { voix indéfinie, masse }\end{array}$ & $\begin{array}{l}\qquad \text { \#on\# } \uparrow \\
\text { résolution des divisions sociales. } \\
\text { Poids de l'opinion publique }\end{array}$ & $\begin{array}{l}\text { rupture d'avec le sens commun, } \\
\text { crise de l'opinion publique }\end{array}$ \\
\hline $\begin{array}{l}\qquad \# \mathbf{l}_{0} \# \\
\text { voix impersonnelle, } \\
\text { « universelle » }\end{array}$ & \begin{tabular}{l}
\multicolumn{1}{c}{ \#il $\# \uparrow \uparrow$} \\
processus d'universalisation \\
d'idées et valeurs
\end{tabular} & \begin{tabular}{|l|}
\multicolumn{1}{|c}{$\# \mathbf{i l}_{\mathbf{0}} \# \times$} \\
processus de « désuniversalisation » \\
d'idées et valeurs
\end{tabular} \\
\hline
\end{tabular}


Pour ce qui est des normes discursives, elles découlent des «valeurs relationnelles intrinsèques » de chacun de ces dispositifs, en ce que ceux-ci non seulement prennent en compte un rapport de forces entre actants plus ou moins symétriques entre eux, mais indiquent aussi une gamme de probables infléchissements du discours.

La configuration \#il\#, pour faire un exemple particulièrement parlant, implique un certain nombre de phénomènes. La non-personne étant un sujet exclu du scénario de communication, on pourrait reformuler \#il\# = («je/tu $)$ «il ». La non-personne ne peut donc pas être sujet d'instance de discours, au contraire elle fait l'objet d'une nominalisation (cristallisation). Par ailleurs, S réalisant \#il\# finira d'abord par impliquer, ensuite par réaliser \#nous\# (éventuellement $\mathrm{S} \equiv \mathrm{M}$, v. supra) moyennant une identification collective de la part des actants concernés : $\mathrm{S}=\#$ je\# et $\mathrm{M}=\#$ tu\#.

La réalisation d'une configuration en implique donc une autre, ou d'autres : si le point de départ est \#vousf\# ou \#vous\#, le « je » (S) peut, sans en avoir l'air, resserrer ou hiérarchiser davantage ses rapports avec cette deuxième personne (singulière ou plurielle), jusque là distante, en dirigeant sa pulsion communicative vers un élément tiers. Il y a là une articulation ultérieure de Ma, l'autre absent : au niveau de $E$, le récepteur à qui s'adresse $\mathrm{Sp}$, d'un côté, et la non-personne évoquée par $\mathrm{S}$ dans le même $\mathrm{D}$, soit au niveau de $\mathrm{E}$, de l'autre. Si la deuxième demeure bien un autre absent, la première aura tendance à se resserrer de la voix énonçante $\mathrm{Sp}$.

Dans le discours politique, la stigmatisation de l'ennemi ou l'insistance sur un péril commun et permanent (parce que cristallisé) peuvent contribuer à rallier le «peuple» à son gouvernement, en estompant encore une fois la distance centre-périphérie, soit \#vous\# > \#il\# > (\#nous $\left.{ }^{\mathrm{f}} \#\right)>$ \#nous\#. C'est la stratégie du bouc émissaire.

Encore, \#il\# est la configuration où, plus qu'ailleurs, la pulsion communicative est orientée vers l'autre absent $\left(\mathrm{M}_{\mathrm{a}}\right)$, même si seule l'analyse détaillée de chaque contexte discursif pourra éclaircir la nature de cette absence. Or sur cette absence peuvent se greffer, pour le meilleur et pour le pire, des valeurs idéales, préconstruites, soit relevant de l'autre latent $\left(\mathrm{M}_{1}\right)$ : la coïncidence morphologique (au niveau de T) bien française entre \#il\# et \#i1 $\#$ en est sans doute la preuve.

Parmi les «effets de discours » qui réaliseraient, sous \#il\#, d'autres configurations, d'abord et surtout \#tu\#, nous nous bornerons à évoquer le phénomène de l'iloiement. Il s'agit d'une véritable mise en scène : le « je » énonçant s'adresse à un «tu » déguisé en « $\operatorname{vous}^{\mathrm{f}}$ » voire en «il » : à l'oral, «qu'est-ce qu'il veut aujourd'hui Monsieur mon mari ? » pourrait exprimer une connivence conjugale (jeu) ou alors une résistance à l'égard d'une mise en relation normale, sorte de pudeur linguistique. Plus en général, il y a tant de façons de mettre le sujet pulsionnel dans une phrase comme : «il fait beau ce matin ». L'écrit écrase cela. L'écrit est de la pulsion versée au silence.

Les capacités humaines de mentir au monde, de tricher, de jouer avec la langue éventuellement pour sortir (Eco, 1979) de son déterminisme (Barthes, 1978) ne peuvent guère être exclues de l'analyse de D. Ces capacités se manifestent évidemment tant à l'écrit qu'à l'oral. Il est pourtant une différence remarquable, concernant le statut de l'interaction: si à l'oral l'interaction en présence fait évoluer D d'après une négociation entre S et M (Kerbrat-Orecchioni, 2000), à l'écrit il se peut bien que la nonprésence de l'autre, régulateur de configuration, augmente les chances de manipulation configurationnelle. Mais cette hypothèse est très difficile à vérifier, car après tout le texte écrit ne peut vivre et fonctionner, agir, qu'à partir de la volonté d'un autre absent, le lecteur - dont, par surcroît, on ne sait presque rien.

\section{Analyse configurationnelle d'un texte écrit}

Pour conclure, nous nous devons d'appliquer la démarche théorique illustrée jusqu'ici : faute d'espace, ce ne sera que l'ébauche d'une analyse configurationnelle d'un document écrit. Cette mise en œuvre - et à l'épreuve - est importante à plusieurs titres : non seulement en ce que, comme nous l'avons dit plus haut, la théorie (notamment du côté de l'articulation des châssis et des dispositifs relationnels) ne pourra 
que gagner en souplesse de ce frottement avec des «textes bien réels »; mais aussi en ce que l'analyse mobilise forcément un élément ultérieur : l'analyste (A), jamais neutre ${ }^{13}$ et, comme nous le soulignions en $\S 2$, seul responsable du déploiement de $\mathrm{T}$ en $\mathrm{D}$. Soit le tract reproduit ci-dessous :

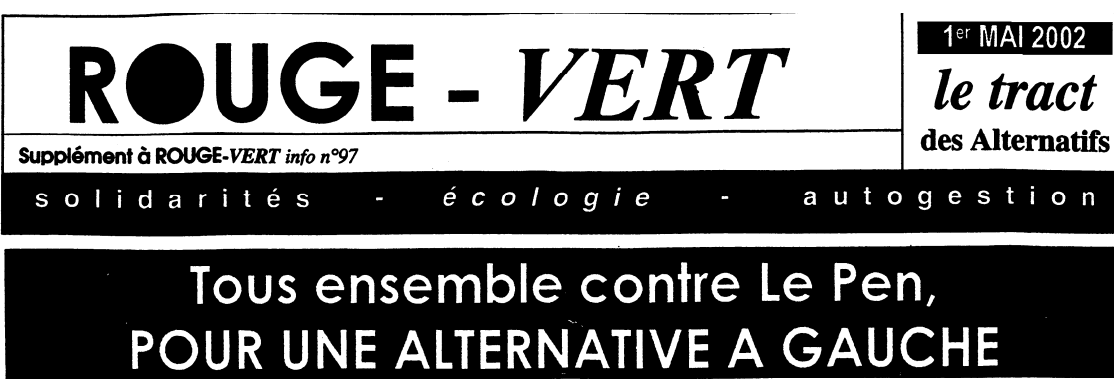

Les résultats du premier tour témoignent d'une situation très grave pour la démocratie, d'une crise profonde de la représentation politique et des institutions de la Vème République.

\section{Le social libéralisme sanctionné}

La politique sociale-libérale du gouvernement a été lourdement sanctionnée par l'électorat populaire. La démagogie sécuritaire de la droite, à laquelle le candidat socialiste a hélas emboîté le pas a mis l'extrême droite en position de force. Nous partageons l'inquiétude et la révolte de la jeunesse, de millions d'électrices et d'électeurs de gauche devant ce gâchis. Le parti socialiste a beau invoquer " un problème de communication ou de pédagogie » ou bien "l'éparpillement des voix sur les petits candidats ». En vérité, c'est sa politique sociale qui a été condamnée : le PARE qui a pénalisé les chômeurs, les 35 heures dévoyées par l'intensification du travail, le refus d'embaucher dans les services publics, les privatisations poursuivies, les menaces sur les retraites, sans parler des provocations d'Allègre dans l'Education nationale. Ce sont aussi ses engagements écologiques non tenus (nucléaire et OGM), son refus de régulariser les sans-papiers, d'abolir la double peine et d'accorder, enfin, le droit de vote aux résidents étrangers qui ont été sanctionnés.

\section{Ecraser les idées de haine}

Aujourd'hui, sans hésitation, il faut faire barrage au candidat de l'extrême-droite raciste et fascisante. Nous devons déıronuer par un vote massit que les idées de haine et vexclusion n'ont pas droit de cité dans notre pays. II ne s'agit en aucune manière d'un ralliement à la droite et à sa politique, mais d'un sursaut démocratique du pays tout entier. Plus le score de Chirac sera élevé et moins il pourra le revendiquer comme un soutien à sa politique partisane. Un faible score pour Le Pen donnera un coup d'arrêt à son arrogance et à la diffusion des idées de haine, de racisme et de discrimination.

\section{Le 1er Mai : Préparer les mobilisations sociales à venir}

Les manifestations unitaires du 1er mai doivent permettre à la jeunesse, aux salariés, à tous les démocrates, d'exprimer avec force leur opposition à l'extrême-droite et leur volonté de défendre les valeurs de la démocratie et de la république. Le Pen écarté, il faudra se rassembler contre l'offensive libérale et cette journée sera aussi un point d'appui pour la défense des droits des salariés, des services publics, des libertés syndicales. De grandes mobilisations sociales se préparent, à la mesure des attaques dont les salaries risquent d'être l'objet.

\section{Construire une alternative à gauche}

Aujourd'hui, il faut non seulement protester, mais s'engager et commencer à construire avec toutes les forces disponibles, une alternative à gauche et un nouveau projet de société.

Dans ce sens, les Alternatifs sont favorables à l'unité d'action de toute la gauche contre la droite et l'extrême-droite. Mais aussi, au sein de la gauche, ils travaillent au rapprochement entre toutes les forces opposées au social libéralisme et qui souhaitent une alternative à gauche, sociale, écologiste et féministe.

\section{Les Alternatifs}

Le mouvement rouge et vert, rouge de la solidarité, vert de l'écologie, Un mouvement de gauche, combatif et unitaire,

pour une gauche vraiment à gauche, pour une gauche vraiment écologiste, pour

l'égalité des droits

Prenez contact : les Alternatifs BP 2016, 06101 Nice cedex 2 email : alternatifs06@wanadoo.fr

Ce document (l'original était en format A4) a été trouvé par hasard sur un banc de la gare de Nice, en 2002, à l'époque des élections présidentielles, et plus particulièrement entre le premier et le second tour, caractérisé par le duel historique (parce qu'inattendu à la veille) entre Chirac et Le Pen. Ce duel surprit 
et fit peur aux Français, c'est pourquoi il rassembla de larges pans de la population, et notamment les jeunes, les étudiants, qui avaient pour la plupart négligé les urnes lors du premier tour.

Notre analyse doit d'abord prendre en compte :

- des éléments circonstanciels, qui définissent le positionnement de l'analyste (A) vis-à-vis de D, puisque tant $\mathrm{A}$ que $\mathrm{S}$ et $\mathrm{T}$ participent de $\mathrm{M}$, ainsi que

- des éléments paratextuels, afin d'établir les châssis relationnels, soit l'expectative configurationnelle que l'on peut légitimement greffer sur T en amont de son analyse microstructurale.

Pour ce qui est des premiers, nous avons déjà dit l'essentiel. La « rencontre » entre S et M, dont $\mathrm{A}$, s'est logiquement produite en absence, mais une absence pour ainsi dire "rapprochée », ce qui est dans la nature même du tract, support qui n'est pas destiné à durer. Mais cette rencontre s'est également produite par hasard: $\mathrm{T}$, qui s'est imposé à $\mathrm{M}$, qui ne le cherchait pas, comme c'était prévu par $\mathrm{S}$ au moment d'actualiser $\mathrm{V}$, est en même temps complètement assujetti à $\mathrm{M}$ - qui pourra le recueillir, éventuellement le lire, ou l'ignorer.

Ces deux observations suggèrent un lien important entre les circonstances de repérage du texte (en absence et par hasard) et ses éléments paratextuels, en gros son « statut » : facilement périssable, le tract doit être consommé rapidement, il se peut donc que le message qu'il véhicule soit un message d'urgence, bien ancré dans l'histoire. En outre, un tract politique échappe aux contrôles, au circuit éditorial, bref au caractère de document officiel : D aura un statut potentiellement subversif, $\mathrm{M}$ sera potentiellement hétérogène, $\mathrm{T}$ sera ponctué d'éléments censés rapprocher $\mathrm{S}$ de $\mathrm{M}$, pour surmonter l'absence de ce dernier. Quant à lui, $\mathrm{S}$ oscille entre sujet présent $\left(\mathrm{S}_{\mathrm{p}}\right)$ et sujet indéfini $\left(\mathrm{S}_{\mathrm{i}}\right)$ : au niveau des châssis relationnels, étant donné l'autre absent $\left(\mathrm{M}_{\mathrm{a}}\right)$, cela correspondrait à un discours à mi-chemin entre le texte d'auteur et le texte publicitaire.

Telles peuvent être les expectatives de A.

En abordant la microstructure, faute d'espace, nous nous bornerons a poser l'accent sur trois fonctionnements que nous estimons particulièrement intéressants et utiles pour mieux illustrer notre démarche d'analyse :

- d'abord le titre, marqué par l'absence de verbe. C'est un véritable slogan : il est donc conforme au châssis relationnel. Qui plus est, les deux lignes dont il se compose évoquent bien une progression, au niveau typographique aussi bien que discursif, que nous pouvons tâcher de représenter à l'aide de nos dispositifs relationnels :

«Tous ensemble $(\#$ on\# $\uparrow)>$ contre le Pen $(\# i l \# \times)>(\#$ nous\# $\uparrow)>$ POUR UNE ALTERNATIVE A GAUCHE $>(\#$ nous $\# \uparrow)>(\#$ ils \#× $) »$

Le groupe se démarque de la masse par la marginalisation d'un tiers, Le Pen; il se précise ensuite par un deuxième démarquage, cette fois-ci par rapport à la gauche gouvernementale : celui qui attaque un groupe distant ne peut être qu'un sujet pluriel qui, ce faisant, renforce son identité. Il y a lieu de penser que cette configuration, par régime de compositionnalité, est la configuration dominante au sein de $\mathrm{D}$;

- la réitération de $\# \mathrm{il}_{0} \# \uparrow$ est aussi remarquable («Aujourd'hui, sans hésitation, il faut faire barrage au candidat de l'extrême droite »; «Le Pen écarté, il faudra se rassembler contre l'offensive libérale »; « Aujourd'hui, il faut non seulement protester, mais s'engager et commencer à construire »; mais aussi les formes infinitives : «Ecraser les idées de haine»; «Préparer les mobilisations sociales à venir»; «construire une alternative à gauche ») : l'effet de discours est la présentation d'un programme d'action ressenti comme un impératif universel, soit $S_{p}>S_{i}>S_{0}$. Les opinions de $S$ ne peuvent, par là, être mises en doute ;

- au niveau de T, M est scindé principalement en deux autres absents $\left(\mathrm{M}_{\mathrm{a}}\right)$, les lecteurs et Le Pen. Alors que celui-ci est stigmatisé par sa nomination-cristallisation $(\# i l \# \times)$, les premiers sont 
« absorbés » par S à travers la réalisation d'un \#nous\# $\uparrow$ englobant (« Nous devons démontrer par un vote massif ») se confondant avec un \#nous\#-auteur ("Nous partageons l'inquiétude et la révolte »).

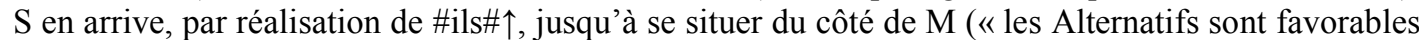
à l'unité d'action de toute la gauche ») : l'effet de discours est $\mathrm{S} \equiv \mathrm{M}$. Le tract se clôture sur le dispositif relationnel annoncé dès le titre : \#nous\# $\uparrow$ par double démarquage (négatif/positif, $\times / \uparrow$ ) par rapport à \#ils\# : la gauche du gouvernement, durement critiquée $(\times)$, d'une part, et le mouvement même, soit S, d'abord indéfini, ensuite impersonnel et finalement nominalisé pour se resserrer $(\uparrow)$, sans en avoir l'air, de ces autres absents censés intégrer le groupe.

\section{Références bibliographiques}

Adam, J.-M. (1999). Linguistique textuelle. Des genres de discours aux textes. Paris : Nathan.

Adam, J.-M. (2005). La linguistique textuelle. Introduction à l'analyse textuelle des discours. Paris : Armand Colin.

Adam, J.-M., Grize, J.-B. \& Bouacha, M. A. (dir.) (2004). Texte et discours : catégories pour l'analyse. Dijon : Editions Universitaires de Dijon.

Agresti, G. (2005). Lingua e Polis. Configurazioni linguistiche e configurazioni sociali nel francese contemporaneo. Préface de Robert Lafont. Rome : Aracne.

Agresti, G. (2008). Les configurations relationnelles pour une didactique plurilingue et pluriculturelle (occitan et français). In Alao, G. - Argaud, E. - Derivry-Plard, M. \& Leclercq, H. (éds). Grandes et petites langues. Pour une didactique du plurilinguisme et du pluriculturalisme. Berne : Peter Lang, 97-109.

Amossy, R. (éd.) (1999). Images de soi dans le discours. La construction de l'éthos. Lausanne : Delachaux \& Niestlé.

Amossy, R. (2000). L'Argumentation dans le discours. Discours politique, littérature d'idées, fiction. Paris : Nathan.

Barthes, R. (1978). Leçon. Paris : Seuil.

Borges, J. L. (1973). Seule la forme peut changer. Le Magazine littéraire, 72, 63-64.

Charaudeau, P. (1995). Rôles sociaux et rôles langagiers. Modèles de l'interaction verbale. Aix-en-Provence : Publications de l'université de Provence, 79-96.

Charaudeau, P. - Maingueneau, D. (dir.) (2002). Dictionnaire d'analyse du discours. Paris : Seuil.

Eco, U. (1979). La lingua, il potere, la forza. Alfabeta, $1^{\mathrm{er}}$ mai.

Grice, H. P. (1957). Meaning. The Phil. Rev., vol. LXVI, 377-388.

Grice, H. P. (1969). Utterer's meaning and intentions. The Phil. Rev., vol. LXXVIII, 147-177.

Grice, H. P. (1977 $)$. Utterer's meaning, sentence-meaning, and word-meaning. In Searle J.R. (éd.). The Philosophy of language, Oxford : Oxford University Press, 54-70.

Guespin, L. (1971). Problématique des travaux sur le discours politique. Langages, 23, 3-24.

Jackendoff, R. (1998). Patterns in the mind, Harvester.

Jakobson, R. (1963). Essais de linguistique générale. Paris : Éditions de Minuit.

Kerbrat-Orecchioni, C. (2000). L'analyse des interactions verbales : la notion de «négociation conversationnelle ». Défense et illustration. Lalies, 20, 63-141.

Lafont, R. (1978). Le Travail et la Langue. Paris : Flammarion.

Lafont, R. (2004). L'être de langage. Pour une anthropologie linguistique. Limoges : Lambert Lucas.

Ricœur, P. (1996). Soi-même comme un autre. Paris : Seuil.

Vygotskij, L. (1934). Myshlenie I rech'. Psihologicheskie issledovanija. Moskvà-Leningrad : Gosu-darvstvennoe social'noèkonomicheskoe izdatel'stvo. 


\footnotetext{
${ }^{1}$ Notre démarche s'inspire principalement de la praxématique de Robert Lafont, et tout particulièrement de sa conception de la langue en tant que lieu d'inscription de la dialectique du même et de l'autre et en tant qu'outil pour la manipulation et transformation de la réalité (Lafont, 1978 et 2004).

${ }^{2}$ Nous ferons ici l'économie des nombreux parasitages ou éléments circonstanciels / subjectifs qui évidemment conditionnent la fabrication d'un texte : compétence linguistique, compétence rédactionnelle (s'il s'agit d'un texte écrit), contexte, etc. Nous nous en tiendrons à une réflexion plutôt générale.
}

${ }^{3}$ On est là dans le sillage de Guespin : « un regard jeté sur un texte du point de vue de sa structuration "en langue" en fait un énoncé ; une étude linguistique des conditions de production de ce texte en fera un discours » (Guespin, 1971 : $10)$.

${ }^{4}$ Les écrivains le savent bien : l'œuvre, une fois achevée, a l'air de bouger toute seule, de «vivre » des lectures diverses que les autres (lecteurs) en font. Les mythes littéraires reposent sur cette variation de la donne discursive : voilà pourquoi ils sont toujours plus ou moins contemporains et se prêtent sans cesse, en diachronie aussi bien qu'en synchronie, à de re-lectures ou à des re-écritures.

${ }^{5}$ «Primitivement et universellement, le sujet n'existe et ne se construit que de sa schise, c'est-à-dire qu'il se fonde sur une part d'autre, qui apparaît très grossièrement dans le $t u$ de la parole solitaire. Dans le dialogue, en disant je, le communicant ouvre la place du $t u$ qu'il instaure en lui à un autre objectif, à une non-personne "prise à partie". Dans $t u$, il est ramené à l'autre du je. Ainsi s'installe une double communication, active-passive, dont, pensons-nous, on ne peut faire fi en aucune analyse» Robert Lafont, in (Agresti, 2005 : 13). On se reportera aux théories de Grice ainsi qu'au «principe d'altérité » de Charaudeau : «il s'instaure [...] entre les deux partenaires un regard évaluateur de réciprocité qui postule l'existence de l'autre comme condition pour la construction de l'acte de communication dans lequel se co-construit le sens» (Charaudeau, 1995). Par ailleurs, «L'interlocution ainsi interprétée se révèle être un échange d'intentionnalités se visant réciproquement. Cette circularité d'intentions exige que soient placées sur le même plan la réflexivité de l'énonciation et l'altérité impliquée dans la structure dialogique de l'échange d'intentions» (Ricœur, $1996:$ 59-60).

${ }^{6}$ L'éthos est évidemment en relation avec les trois dimensions de l'altérité. En particulier, la notion d'« éthos préalable» ou «prédiscursif» est essentiellement liée à l'autre latent, réservoir de valeurs et d'images standardisées. Cf. Adam (1999), Amossy (1999 et 2000).

${ }^{7}$ Remarquons que, dans ces deux exemples, le texte est fabriqué d'après un modèle oral, comme pour recréer (inconsciemment ?) un contexte in praesentia.

${ }^{8}$ Cette influence est sans doute la même qui pousse les gens à s'habiller plus ou moins tous pareils, à faire les mêmes gestes, bref à se ressembler et à se conformer aux usages de l'endroit. Cette doxa où le sujet peut s'engouffrer et sa subjectivité disparaître n'est donc guère une force, une contrainte exclusivement linguistique, il s'agit bien d'une sémiotique. Mais à travers le texte elle est bien lisible, voire mesurable.

${ }^{9}$ Une réflexion ultérieure pourra prendre en compte également la communication médiatisée par ordinateur (CMO).

${ }^{10}$ Pour une synthèse portant sur les différents emplois de ces notions, on se reportera à Charaudeau - Maingueneau (2002: 221-223, 228-231).

${ }^{11}$ Dans cette perspective, les langues régionales ou minoritaires présentent le plus grand intérêt, à cause de la connotation, souvent remarquable, de M, par rapport au maillage social habité par une langue d'Etat.

12 Pour des raisons techniques liées au formatage du présent texte, on renonce ici à employer la police de caractère créée exprès pour représenter nos dispositifs relationnels. Dans le Tableau 4 on les a donc explicités. 
13 C'est une des leçons les plus fascinantes qui nous viennent de la physique quantique : l'expectative de l'observateur influe sur le comportement des quantons observés... Comment l'analyste de textes pourrait-il sortir de l'idéologie qui le gouverne? 